\title{
Bericht über die bei der deutschen Tiefsecexpedition beobachteten Viggel.
}

Von Dr. E. Vanhöffen, Kiel Zool. Institut.

(Hierza eino Karto.)

Während der Fahrt der Valdivia, die den Atlantischen Ocean von $62^{\circ} \mathrm{N}$. Br. bis $58^{\circ} \mathrm{S}$. Br. durchfuhr, dann der Eiskante des antarktischen Meeres vom 7. bis zum 63. Längengrad folgte und schliesslich den indischen Ocean der Länge und Quere nach durchmass, auch an selten von Zoologen besuchten Küsten landete, schien es von Wert, tägliche Aufzeichnungen über das Erscheinen der Vögel zu machen. Einer Aufforderung von Herrn Professor Reichenow folgend, der die von uns mitgebrachten Bälge bestimmte, ${ }^{1}$ ) erlaube ich mir diese Beobachtungen hier mitzuteilen.

Den ersten grossartigen Eindruck von der reichen Vogelwelt des Meeres erhielten wir am Nachmittag des 3. August 1898 bei der Annäherung an die schottische Kinste, als wir am Eingange zum Firth of Forth nahe am Bass Rock, der wichtigsten Brutkolonie des Tölpels, Sula bassana, vorüberfuhren. Wie ein gewaltiger Kasten mit niedriger dachförmiger Kuppe hebt sich der allseitig steil abfallende Fels aus dem Meer. Nur an den steilsten Abstürzen war unter dem grünen Dach, das kurzer Rasen bedeckte, noch die dunkle Farbe des Gesteins erkennbar, im übrigen erschienen seine Seiten weiss von den in langen Reihen auf allen Vorsprüngen dicht über einander sitzenden Vögeln. Ein Schuss, der die Vögel nur erschrecken sollte, störte viele Hunderte von ihnen auf, ohne dass ihre Reiben gelichtet erschienen. Die Tölpel beherrschen den Fels; neben ihnen wurden nur in geringer Menge noch die beiden Lummen Uria grylle und Uria lomvia sowie die dreizehige Möve Rissa tridactyla bemerkt.

Als wir dann an 5. August längs der Ostküste Schottlands nach Norden dampften, erschien ein Steinschmätzer, Saxicola oenanthe*, an Bord, und am 6., nachdem Foul Island passiert war, zeigten sich die ersten Eissturmvögel oder Mallemucken, Fulmarus glncialis, beim Schiff. Angesichts Far Öer und bei

1) Die Vogel, deren Bălge Herrn Professor Reichenow vorgelegen haben, sind nit einem * bezeichnet. 
der Rundfahrt um Suderö wurden dunkle Lummen, Uria arru(?) mit ihren Jungen angetroffen und Papageitaucher, Mormon fratereula, die über die Wellen hüpfend, sich aus dem Bereich des Schiffes zu retten suchten. Auch einige Kormorane, Phalacrocorax carbo, und die Mantelmöve, Larus marinus, liessen sich sehen ausser den Eissturmvögeln, die dem Schiff folgten und in kleinen Colonien die Steilwände der Ufer bewohnten. Auch am 9. und 10. August auf hoher See im Westen von Schottland begleiteten uns Fulmare noch bis $55^{\circ} \mathrm{N}$. Br., dann wurden sie nicht mehr gesehen. Dafür fand sich nach stürmischer Nacht und bei unruhiger See am 12. August westlich von Irland ein anderer dunkler Sturmvogel ein, mit weisser Unterseite, den ich für einen Puffinus hielt. Am 13. wurden mehrere Exemplare derselben Art zwischen den noch hochgehenden Wellen hinschwebend bemerkt.

Nachdem sich die See etwas beruhigt hatte, stellten sich etwa unter $45^{\circ} \mathrm{N}$. Br. die kleinen Sturmschwalben, Oceanites oceanicus, ein, die, abgesehen von unserem Aufenthalt bei den Kanaren (vom 20.-23. August), täglich vom 15. bis zum 25. August, bis $25^{\circ} \mathrm{N}$. Br., beobachtet werden konnten.

Auf den Kanaren fielen bei der Fahrt von Orotava nach Icod nur ein Raubrogel und im Hafen von Las Palmas grosse Blaumöven, Larus glaucus (?), auf. Während wir dann an der Westküste Afrikas in der Richtung von Kap Bojador nach Boavista entlang fuhren, kamen mit einem Schwarm kleiner Nachtfalter zahlreiche Landvögel an Bord. Ein am 24. August gefangener Singvogel wurde provisorisch als Acrocephalus bestimmt und an demselben Tage soll sich ein Wiedehopf gezeigt haben. Am 25. wurden nur Petersvögel, Oceanites oceanicus*, gesehen, am 26. aber waren wieder Landvögel vorhanden, unter denen wir eine Bypolais zu erkennen glaubten. Die Zahl der Landvögel war am. 27. und 28. August besonders reich, nachdem am 26. der Passat an der Grenze des Guineastroms eingesetzt und einen Seegang von Stärke 6 erzeugt hatte. Mit Sicherheit konnte nur Lanius senator* bestimmt werden; ausser ihm glaubten wir noch die vorhererwähnten Acrocephalus und Hypolais zu erkennen, ferner einen grossen Vogel, der an einen Kukuk oder kleinen Raubvogel erinnerte, aber nicht genau beobachtet werden konnte. Wie es schien, zogen alle diese Vögel mit uns den Kap Verdeschen Inseln zu, die am 29. Morgens in Sicht kamen. Am 
31. August zeigte sich im Süden dieser Inseln über einer Heerde von Zahnwalen, ruhelos hin und her fliegend, ein einsamer dunkelbrauner Vogel mit weisser Unterseite, der mir ein Puffoms zu sein schien. Auch am 12. August wurde dieselbe Art in Gesellschaft von Delphinen bemerkt. Es sind weitere Beobachtungen darüber nötig, ob hier ein zufälliges oder ein regelmässiges Zusamment reffen vorliegt.

Auf der Fahrt von $10^{\circ} \mathrm{N}$. Br. bis zum Äquator, parallel der afrikanischen Küste, vom 2. bis 8 . September, wurden keine Vögel bemerkt; am 9. aber fielen bei etwa $1^{\circ} \mathrm{N}$. Br. und $3^{\circ} \mathrm{W}$. L., an der Grenze zwischen Südäquatorialstrom und Guineastrom, kleine Sturmschwalben, Oceanodroma cryptoleucc*a, auf, die ich mit ausgebreiteten Fliggeln auf dem Wasser laufen sah. Ann Abend hörten wir wiederbolt ihren Schrei: "Kerr $\mathrm{kwi}$," wenn sie sich den erleuchteten Fenstern näherten, oder um die Laternen am Mast flatterten, und am 12. wurde einer dieser St. Petersvögel gefangen, der durch die offenen Fenster in den Salon geflogen kam. Sie begleiteten das Schiff noch einige Tage, wurden aber am 13., als wir uns der Kamerunbucht näherten, nicht mehr gesehen. Am 9. soll sich zum ersten Male ein Tropikvogel Phaeton gezeigt haben.

Vom 15. bis 25. September waren wir in Kamerun. Der auffallendste Vogel ist dort Gypohierax angolensis*, der Geieradler, der häufig im Mangrovegebiet zu beobachten war, doch kamen nur junge, noch nicht ausgefürbte Vögel zum Schuss. Ausser ihm wurden dort noch Charadrius tenellus, ${ }^{*}$ Barbatulu subsulphurea,* Didua principalis,* Spermestes poensis* und zwei Webervögel, Ploceus nigerrimus* und Ploceus collaris*, erlegt, die an den Blättern der Ölpalmen ihre Nester aufhängen und das Material für ihr Flechtwerk aus den zerzupften Fiederblättchen gewinnen. Solche zerzausten Palmen mit Weberkolonieen fanden sich nicht selten bei Victoria und an den Ufern des Wuri bei der Fabrt nach Jambassi. Sonst wurden bei dieser Fahrt noch graue Papageien, Eisvögel, Tauben, sogenannte „Wurivögel," wohl Wasserhühner mit langen, roten Beinen und schöne weisse Reiher gesehen.

Zwischen Kamerun und der Kongomündung sahen wir keine Vögel. Auf dem $\mathrm{Kong} 0$ und an seinen von Mangrove und Schilf bewachsenen Ufern zeigten sich schwalbenähnliche Vögel, der Schattenvogel Scopus umbretta und Eisvögel, Ceryle 
rudis, die, in schnellem Fluge dicht über dem Wasser hinschwebend, von einem Ufer zum andern eilten, ein grosser, grauer Reiher, ein storchartiger Vogel, vielleicht Mycteria senegalensis (?), der Schildrabe, Corvus scapulatus, mit weissem Brustring und in der Nühe von Boma ein grosser Flug Seeschwalben Sterna sp. Erlegt wurden dort Gypohierax angolensis, der Geieradler, ebenso wie in Kamerun nur in jungen Exemplaren, die sich hauptsächlich von Krabben und Einsiedlerkrebsen zu ernähren scheinen, Anhinga rufa der Schlangenhalsvogel, den wir geschickt tauchen oder mit ausgebreiteten Flïgeln am Ufer sitzend sich Kühlung zufächeln sahen, und der zierliche Silberreiher, Ardea gargetta. Bei Boma bemerkten wir eine Nachtschwalbe (?), die aus dem dichten Savannengras gerauschlos aufflog, kleine, einem Rotschwänzchen äbnliche Singvögel und den Schildraben, und bei Banana wurden Passer diffusus* und Turdus bocngei* erbeutet. Am 5. Oktober morgens lichteten wir in Banana die Anker, um in studlicher Richtung zur grossen Fischbai zu dampfen. Bei dieser Fahrt wurden nur am 8. und 9. Oktober kleine St. Petersvögel gesehen, die nicht mit Sicherheit bestimmt werden konnten. Es ist sehr wahrscheinlich, dass auch hier diese Vögel die reiche Ansammlung pelagischer Tiere an einer Stromgrenze ausbeuteten.

Am Tage darauf erreichten wir die grosse Fischbai, die von völlig vegetationslosen Sanddünen umgeben ist. Sie gab uns zum zweiten Male Gelegenheit, eine grossartige Vogelwelt zu studieren und gleichzeitig die gewaltige Produktionskraft des Meeres zu bewundern. Denn das Meer ernährt hier Tausende grosser gefrässiger Vögel, von denen nicht wenige Arten hier ihre Nordgrenze erreichen. Schon bei der Einfahrt in den Hafen waren wir erstaunt, ähnliche Arten wie bei Schottland und den Far Öer zu erkennen. Wir sehen dort den südlichen Tölpel, Sula capensis*, senkrecht in's Wasser herrabstossend fischep. Erst lässt sich der Vogel mit etwas ausgebreiteten Flügeln fallen, dann legt er plötzlich die Flügel dicht an und saust wie ein Pfeil in's Wasser herab. Ferner sassen ganze Scharen dunkler Kormorane, Thalacrocorax capensis*, nebeneinander aufgereiht am Strande, und in graziösem Fluge schwebten Seeschwalben und die Sturmvögel, Puffinus und Procellaria aequinoctialis*, über dem Wasser. Ein dritter Sturmvogel, die Kaptaube, Daption capense, fiel durch ihr buntes, schwarz und weiss geflecktes 
Gefieder auf. Im innersten Teile der ausgedehnten Bucht machten sich Flamingos, Thoenicopterus roseus, bemerkbar, die in langen Reihen am Strande zu exercieren schienen, fischend im Wasser standen oder plötzlich auffliegend sich als rosenrote Wolke erhoben, und Pinguine, Spheniscus demersus ${ }^{*}$, von denen wir nur wenige, noch nicht völlig ausgewachsene Exemplare bemerkten. Auf der flachen, die Bucht absperrenden Tigerhalbinsel tummelten sich zahlreiche Strandläufer. Dort gelang es, zwei Exemplare eines neuen Regenpfeifers, Charadrius rufocinctus ${ }^{*}$, neben dem bekannten Charadrius hiaticuld ${ }^{*}$ zu erlegen und am Ende der Bucht am Fusse hoher Dünen zeigten sich Schildraben, Corvus scapulatus, die durch Reste getrockneter Fische angelockt waren.

Als wir am 12. October die Fischbai verliessen, kamen wir in stürmische See. Draussen erschienen am 13. wieder St. Petersvögel, die in der Bucht nicht bemerkt waren, ferner folgten Daption capense, Procellaria aequinoctialis und der gelbschnäblige Albatross, Thalassogeron chlororhynchus, der hier zum ersten Male sich zeigte, dem Schiff. Am 14. wurde nur noch dieser und dazu zum ersten Male der grosse Albatross, Diomedea exulans, von uns gesehen. Während dann vom 15.-17, Oktober alle Vögel fehlten, gelang es am 18. einen grossen Albatross von $2,90 \mathrm{~m}$ Flügelspannung mit schwarzen Flügelspitzen, grauem Anflug. am Halse, blaugrauen Füssen und gelblichen Schnabel zu angeln. Auch Procellaria aequinoctialis erschien. Am 20, wurden Procellaria aequinoctialis und Diomedea exulans*, am 23. P. aequinoctialis und Daption capense beobachtet. Am 25. Oktober näberten wir uns bereits dem Kaplande, doch war das Land noch nicht in Sicht, als wir in einer Tiefe von etwa $3000 \mathrm{~m}$. unsere Netze auswarfen. Bei dem stillliegenden Schiff fand sich hier eine grössere Anzahl von Vögeln ein, die sich mit Geschrei und Gepiepse um die mit einer Angel ausgeworfenen Speckstückchen stritten. Am eifrigsten war Procellaria aequinoctialis dabei, die selbst den kleinen Albatrossen, Diomedea melanophrys, den Köder wegzunehmen versuchten und die kleineren Kaptauben und St. Petersvögel verdrängten. Die Albatrosse waren vorsichtiger. Mit schnarrendem, wie „karr" klingendem Ruf schafften sie sich Platz, doch fassten sie den Köder nur mit der Schnabelspitze und liessen beim Anziehen der Angel los, während die braunschwarzen Procellarien mit grünlichem, schwarz geflecktem Schnabel, 
schwärzlichen Füssen und weissem Kinn sich leicht fangen liessen. Auch 2 Fxemplare der Kaptauben wurden genngelt. Vor Kapstadt gesellten sich am 26. Oktober zu Proeellaria aeguinoctialis und Daption capense noch zahlreiche Mantelmöven, Larus dominicanus, hinzu, die uns früher nicht begegnet waren. An demselben Tage noch setzten wir unsere Fahrt nahe an der Südküste Afrikas fort. Dort wurde am 27. zwischen dem Kap der guten Hoffnung und Kap Agulhas nur Procellaria aequinoctialis, am 28. in der Plettenberg-Bucht nur Diomedea exculans, am 29. in der Francis Bai Larus dominicanus, Procelluria aequinoetialis, Diomedea exulans, Daption capense und St. Petersvögel, wohl Oceanites, und in der Algoa Bai bei Port Elisabeth am 31. Oktober Larus dominicanus angetroffen.

Da wir uns bei der Rückfahrt von Port Elisabeth nach Kapstadt vom 1. bis 5. November etwas ferner vom Lande hielten, um auch grössere Tiefen im Gebiet des Agulhas-Stroms zu erforschen, so waren keine Dominikanermöven zu sehen. Es zeigten sich nur die 3 Albatrossarten Diomedea exulans, $D$. melanophrys* und Thalassogeron chlororhynchus. Besonderes Interesse erregte am 1. November südlich von der Algoa Bai der erste Riesensturmvogel, Ossitraga gigantea, der sich furchtlos dicht beim Schiff niederliess und durch einen Schuss erlegt wurde, da er auf die ihm zugeworfene Angel nicht reagierte.

Im Hafen von Kapstadt wurden nur Dominikanermöven, dann bei der Ausfahrt am 13. November Procellaria aequinoctialis, Daption capense, Diomedea exulans und kleine Pinguine, Spheniscus demersus, bemerkt.

Das reiche Vogelleben bildet ein Gegenstück zu den Vogelkolonien an den nordeuropäischen Küsten. Während in den warmen Meeren von $50^{\circ} \mathrm{N}$. Br. bis $15^{\circ} \mathrm{S}$. B. nur St. Petersvögel, Puffinus und der Tropikvogel erschienen, finden wir die nördlichen und südlichen kalten Gewässer von Tölpeln und Cormoranen belebt und anderen gefrässigen Fischern, da den Fulmarus glacialis des Nordens die Procellaria aequinoctialis des Südens, den Alken und Lummen die Pinguine und der Mantelmöve des Nordens die Dominikanermöve des Südens entsprechen. Dass das vogelarme Meeresgebiet so ungleich nördlich und südlich vom Aequator verteilt ist, beruht auf dem weiten Vordringen des Golfstroms, der die atlantische Küste Europas und des kalten Benguelastroms, der die Westküste Südafrikas bespült. 
Als wir dann zur Aufsuchung der Bouvet-Insel nach Sidden dampften, wurden vom 14. bis 16. November keine Vögel gesehen. Am 17. aber beim Eintritt in die Westwindtrift erschienen wieder Vögel als Vorboten einer neuen Region. Bereits am Morgen umkreisten uns viele Exemplare von Prion coeruleus, eines kleinen, oben graublauen, unten weissen Sturmvogels mit dunkler Schulterbinde, und mehrere kleine Albatrosse, Dionedea melanophrys. Nur die letzteren kamen in die Nähe des Schiffes, wïhrend die ersteren sich durch unser Fischen nicht in ihrem heftigen Hin- und Herfliegen stören liessen. Am Tage darauf wurden Diomedea exulans und $D$. melanophrys von folgenden Abmessungen geangelt:

$\begin{array}{lcc} & \text { Schnabelspitze - Schwanzspitze. } & \text { Flügelspannung. } \\ \text { D. exulans } & 1,35 \mathrm{~m} & 3,12 \mathrm{~m} . \\ \text { D. melanophrys } & 0,95 \mathrm{~m} & 2,14 \mathrm{~m} .\end{array}$

Der Schnabel von $D$. melanophrys war gelbgrün, vorn dunkler bis rötlich braun, der Fuss bläulich fleischfarben. Ferner zeigte sich ausser Prion coeruleus noch die letzte Procellaria aequinoctialis.

Besonders interessant in ornithologischer Hinsicht war der 19. November, der unserer Sammlung 3 neue Sturmvögel brachte: Aestrelata mollis* und Priofinus cinerens*, die nur dieses einzige Mal in je einem Exemplar beobachtet werden konnten, und einen St. Petersvogel Cymodroma melanogaster*. Sonst begleiteten noch ein Albatross, $D$. exulans, und mehrere Prion coeruleus das Schiff.

Nachdem dann am 20. das Barometer um $22 \mathrm{~mm}$ gefallen war, erschien bei schwerer See und Windstärke 10 zum ersten Male der braungraue Albatross, Phoebetria fuliginosa, der durch die weissen Augenlider charakterisiert ist, und der erste antarktische Pinguin, Pygoscelis antarctica. Am 21. zeigten sich nach Südsturm bei noch sehr hoch gehenden Wellen wieder zahlreiche Vögel: Phoebetria fuliginosa, Prion coeruleus, Cymodroma melanogaster, Priocella glacialoides, eine neue Erscheinung, und Daption capense, die wir seit dem Tage unserer Abfahrt von Kapstadt den 13. November nicht mehr gesehen hatten. Am 22. November bei verhältnismässig ruhiger See und auch am 23., als die See nach unruhiger Nacht, in der beigedreht werden musste, noch recht hoch ging, wurden keine Vögel bemerkt. Am 24. aber fanden sich ausser Daption capense und Phoebetria fuliginosa zahlreiche Exemplare von Priocella glacialoides und 
eine Schar von Pinguinen ein, Pygoscelis antarctica, kenntlich an der schmalen schwarzen Kehlbinde. Das Auftreten eines Schwarmes dieser nicht fliegenden Vögel, die wie Delphine tiber die Wellen hüpfen und mehr unter als uber dem Wasser zu schwimmen scheinen, wurde zusammen mit dem Erscheinen zahlreicher Kaptauben und Sturmvögel als Anzeichen nahen Landes gedeutet. In der That kam am 25. Mittags, nachdem wir das erste Eis passiert hatten, schon die Bouvet-Insel in Sicht, die Kaptauben, Daption capense", Priocella glacialoides*, Cymodroma melanogaster, Prion coeruleus und Phoebetria falliginosa umschwirrten. Unter ihnen fielen als neue Ankömmlinge eine weisse Eismöve, Pagodroma nivea, und die grosse Ranbmöve, Lestris antaretica a auf. Eine Landung auf der Insel war nicht auszuführen. Am 28. wurden bereits mehrere Eismöven bemerkt und am 29., als wir unseren Kurs im Süden der Bouvetinsel fortsetzten, kamen zu den bereits an den vorhergehenden Tagen bemerkten Arten wie Prion coernleus*, Pagodroma, Pygoscelis antarctica ${ }^{*}$, Daption, Phoebetria fuliginosa* und Priocella noch Ossifraga gigantea*, der Riesensturmvogel, hinzu, der seit der Agulhasbank gefehlt hatte, und ein neuer Sturmvogel Thalassoeca antarctica, der ebenso sicher wie Pagodroma nivea die Nähe des Eises verkündet. Mehrere Riesensturmvögel wurden auf einem der Eisberge ruhend angetroffen.

Am 30. November erreichten wir den südlichsten Punkt im Gebiet des atlantischen Oceans, da uns das Treibeis am weiteren Vordringen hinderte. Bis zur Eisgrenze begleitete uns nur Pygoscelis antarctica, Pagodroma nivea und Thalassoeca antarctica. Es lassen sich demnach im suddlichsten Teil des atlantischen Oceans auch 2 Gebiete unterscheiden, die durch die Vögel der Westwindtrift, Cymodroma melanogaster, Prion coeruleus und Phoebetria fuliginosa, und die Vögel der Eiskante Pygosselis antarctica, Pagodroma nivea und Thalassoeca antarctica characterisiert werden können.

Dem Eise ausweichend nahmen wir unter $57^{\circ} \mathrm{S}$. Br. und $8^{\circ}$ O. L. einen östlichen Kurs auf. Am 1. Dezember war das Packeis verschwunden, nur einzelne Eisberge rauschten vorüber. Zwischen ihnen fiel durch breiteren bläulichen Schnabel, dunkel gesäumten Schwanz und mehr graues Gefieder eine neue Prionart auf, die als Prion desolatus* bestimmt werden konnte. Ferner zeigten sich noch Prion coeruleus*, Ossifraga gigantea, Cymodro- 
ma melanogaster, Pagodroma nivea und Thalassoeca antnretica.* Am 2. Dezember wurde Sterna macrura* völlig erschöpft, aber noch lebend, mit leerem Magen an Bord gefunden und ein zweites Exemplar derselben Art in schnellem Fluge boch über dem Schiff hinziehend gesehen. Sonst erschienen, da wir immer wieder in das Packeis hineinkamen, nur noch Pagodroma und Thalassoeca.

Da wir uns nun, um vom Eise frei zu kommen, nach Norden wandten und so eisfreies Wasser erreichten, in dem nur noch einige Eisberge sichtbar blieben, zeigten sich am 3. wieder Prion coeruleus und $\boldsymbol{P}$. desolatus, Thalassoeca antarctica und die Kaptaube, die an den beiden letzten Tagen gefehlt hatte. Am 4. Dezember stellte sich dazu noch Priocella glacialoides ein, und Pygoscelis antarctica wurde nahe am Schiff vorbeischwimmend und auf einem Eisberg ruhend bemerkt.

In der Nacht trafen wir wieder auf Eis. Am Morgen des 6. im eisfreien Meer folgten 9 Exemplare von Phoebetria fuliginosa* ferner Prion coerulens und $\boldsymbol{P}$. desolatus und Daption capense dem Schiff. Fünf Pinguine, Pygoseelis antarctica, die vorbei trieben, machten sich durch Rufe bemerkbar. Auch ein St. Petersvogel wurde gesehen. Thalassoeca und Priocella, die am Tage fehlten, erschienen am Abend und kündigten Eis an, das sich in der Nacht denn auch einstellte. Am 6. begegneten wir wieder einem Eisberg mit Pinguinen, und ausser zahlreichen Taubensturmvögeln, Prion, wurden mehrere Albatrosse, Phoebetria, und Kaptauben, sowie ein Riesensturmvogel und eine Priocella glacialoides beobachtet. Da wir uns nun einer Gegend näberten, in der das Eis weniger weit nach Norden vordringen sollte, fuhren wir in südöstlicher Richtung, um weiter nach Süden zu kommen. Bei ziemlich blauem ruhigen Meer zeigten sich am 7 . einige kleine Eisberge am Horizont und nur wenige Vögel. Prion und Yhoebetria waren sichtbar. Am 8. stellte sich wieder eine grössere Anzahl von ihuen ein. Prion banksi*, ein vorher bei der Expedition nicht beobachteter Taubensturmvogel, wurde erlegt. Rrion coeruleus und 2 . desolatus umflogen in grösserer Anzahl während des Fischens das Schiff. Während sie sonst, so oft wir die Taubensturmvögel beobachtet hatten, stets in hastigem, ruhelosem Fluge vorüber eilten, setzte sich hier einer derselben für einen Moment aufs Wasser, um ein rotes Flickchen aufzupicken, das von einem unserer Netze herabfiel. Sonst fanden 
sich noch mehrere andere braune Albatrosse, von denen einer einen helleren Rücken als die tabrigen hatte, ein Lxemplar von Thalassoeca antaretica und ein St. Petersvogel, wohl Oceanites oceanicus, bei uns ein.

Zu den genannten Arten gesellten sich am 9. noch Daption capense, und nachmittags kamen mehrere Exemplare von Pagodromu nivea und Thalassoeca antarctica hinzu. Bald darauf erschienen schon einige Eisstücke und abends musste des Eises wegen nach Norden gewendet werden. Am 10. wurden bei ruhiger, eisfreier See eine Phoebetria, ein Oceanites und eine Thalassoeea bemerkt. Am Abend aber kamen wir wieder in's Eis. Ausnahmsweise fehlten hier auch während der hellen Nacht die beiden, sonst so charakteristischen Sturmvögel im Eise. Es waren am 11. Dezenber nur zwei Daption, eine Phoebetria, einige Prion, ein Oceanites und in der Ferne ein anscheinend verirrter, weisser Albatross, wohl Diomedea melanophrys, sichtbar. Am 12. blieb uns nur Prion desolatus treu, die übrigen Vögel hielten sich fern bei Schneetreiben und stürmischem Wetter. Am 13. kamen Daption und Ploebetria hinzu, am 14. wurden nur wenige Exemplare von Thalassoeca und am 15. Phoebetria fuliginosa auf kleinen Eisbergen ruhend bemerkt.

Am südlichsten Punkt, den wir erreichten, bei $64^{\circ} 14^{4}$ S. Br. und $53^{\circ} 30^{\prime}$ O. L., 104 Seemeilen von der für EnderbyLand angegebenen Position, gab es wieder eine neue Vögelversammlung. Da wir des dichten Eises wegen umkehren mussten, konnten Thalassoeca und Pagodroma nicht fehlen. Ausser ihnen hatten sich Phoebetria, Priocella, Daption, Prion desolatus und Oceanites oceanicus* eingefunden. Dieselbe Gesellschaft folgte auch noch am 17. dem Schiff. Am 18. und 19. Dezember nahmen wir Abschied von den Vögeln des äussersten Südens. Am 20. erschien Diomedea melanophrys und am 21. traten bereits Diomedea exulans, Thalassogeron chlororhynchus und Procellaria aequinoctialis auf. Am 22. Morgens wurde Sterna macrura* an Bord gefangen. Ferner zeigte sich $D$. exulans, Phoebetria, Ossifraga, Procellaria aequinoctialis und Prion coeruleus und am 23. und 24. wurden $D$. exulans, $P$. aequinoctialis, Daption, Prion coeruleus und Sterna macrura gesehen.

Obwohl wir während der Fahrt durch das antarktische Meer an reiches Vogelleben gewöhnt waren, wurden wir doch überrascht durch den Reichtum an Arten und Individuen und 
durch die Harmlosigkeit und Furchtlosigkeit der Vögel auf den Kerguelen, die wir am 25. Mittags erreichten. Mit dem Vernglas sahen wir schon im Vorbeifahren an der Küste der am weitesten nach Osten vorspringenden Halbinsel brütende Albatrossse und Pinguincolonien. Dann machten sich besonders die Comorane, Phalacrocorax verrucosus ${ }^{\ddagger}$, bemerkbar, die einzeln oder in kleinen Flügen mit lang vorgestrecktem Halse niedrig über das Schiff hinwegstreichend dem Lande zustrebten. In dichten Scharen schwärmten vor der Einfahrt zum Gazellebafen Prion und Oceanites über den flutenden Büscheln des Riesentangs Macrocystis zahlreiche Kaptauben, Phoebetria, und die grosse Raubmöve, Lestris antarctica, sowie einige Riesensturmvögel folgten dem Schiff. Bald nachdem der Anker im Gazellehafen gefallen war, wurden die Pinguine, die interessantesten gefiederten Bewohner der Insel, besucht. In den Nischen zwischen schiefkantigen abgestürzten Blöcken, die sich an steiler Wand aufgetürmt haben, hausten zahlreiche Pärchen von Catarractes chrysocome, dem goldhaarigen Pinguin, den wir schon vorher in langen Zügen durch die Wellen tauchen gesehen hatten. Sie liessen sich im Bewachen ihrer grìnlichweissen bis braungelben Eier durch die Besucher nicht stören, steckten nur verwundert schnatternd die Köpfe zusammen. Ebenso wenig kümmerten sich die weissen Scheidenschnäbel, Chionis minor*, um den Besuch, die frech berbeikamen, um Pinguineier zu rauben und sich selbst mit den Händen fangen liessen. Erstaunt über die fremden Eindringlinge zeigte sich die grosse Raubmöve, die jedes von uns angelegte Depot untersuchte und kaum aufflog, wenn man sie verjagen wollte. Wenig scheu war auch Sterna virgata*, die zierliche Seeschwalbe der Kerguelen, und ein kleiner St. Petersvogel, der an den Abhängen im Innern der Insel schwalbenähnlich umherflog, was eigenartig berührte, da man ihn sonst nur auf hoher See anzutreffen gewohnt ist. Unsicher scheinen sich die kleinen Enten, Anas eatoni*, zu fühlen, die sich an den meisten kleinen Seen und Tümpeln und an der Mündung eines Baches zeigten. Nur ein Vogel war so scheu, dass es uns nicht gelang, ihn zu erbeuten, Pelecanoides urinatrix. Kaum waren wir ihnen auf Schussweite nahe, so erhoben sich die kleinen schwarzen Vögel und suchten, im Fluge an die Teiste oder Krabbentaucher erinnernd, das Weite. Es wurde allerdings auch kein ernstlicher Versuch gemacht, sie zu verfolgen. Von bäufigeren Vögeln ist dann noch die Mantelmöve, Larus domini- 
canus $^{*}$, zu erwähnen, die besonders auf kleinen, mit üppiger Vegetation bedeckten Inseln brïtete, aber dort auch ihre Eier vor den zudringlichen Scheidenschnäbeln schützen musste. In grosser Schar fanden sie sich mit Ossifragn gigantea und den Raubmöven bei den blutigen Körpern der erlegten und abgehäuteten Seeelephanten ein. Besonders die Riesensturmvögel waren durch ihre kräftigen Schnäbel geeignet, die Cadaver zu öffnen, zu zerfleischen und für die kleinen Vögel zugünglich zu machen. Bald hatten sie sich so voll gefressen, dass sie kaum noch zu fliegen im Stande waren.

Nur in einem Exemplar wurde am Gazellehafen Pygoscelis forsteri, der Königspinguin angetroffen, der, als er sich verfolgt sah, erst aufgerichtet, dann auf allen Vieren laufend, das Wasser zu gewinnen suchte, während wir dort den nach seinem Geschrei benannten Eselspinguin, Pygoscelis papua, in 2 Exemplaren erhielten. Im Weihnachtshafen, wo wir, kurz bevor wir die Insel verliessen, am 29. Dezember, anlegten, wurden mehrere Königsund Eselspinguine lebend an Bord gebracht. Am 30. Dezember erschienen bei stürmischem Wetter Dionedea melanophrys und Procellaria aequinoctialis beim Schiff und zum letzten Male zeigte sich Prion coeruleus als letzter Vertreter der antarktischeVögel.

Wenn wir nun auf die Verteilung der antarktischen Vögel, wie sie sich nach unsern Beobachtungen darstellte, zurückblicken, so zeigt sich, dass Daption capense in dem von uns befabrenen Gebiet die weiteste Verbreitung hat. Sie wurde im atlantischen Ocean von der grossen Fischbei unter $16^{\circ} \mathrm{S}$. Br. bis zum Cap, dann wieder von $47^{\circ} \mathrm{S}$. Br. bis zum Packeise im Süden und im indischen Ocean von der Eiskante bis zu den Kerguelen angetroffen. Indessen hat es den Anschein, als ob die südafrikanische Daptioncolonie keine direkte Verbindung mit der Bouvet-Insel und dem südlichen Eise hat, da auf der ganzen Strecke vom 14-21 November Kaptauben fehlten. Sie scheinen demnach keine besonders guten Flieger $z u$ sein und sich meist nahe am Lande oder am Eise zu halten, wo sie ausruhen können. Das gilt in noch höherem Grade für Ossifraga gigantea, der ein träger Vogel ist und am Cap, dann ganz nahe der Bouvet-Insel, auf Eisbergen ruhend und bei den Kerguelen gefunden wurde. Interessant ist ferner die Verbreitung der Prion-Arten. Sie gingen nach Norden nicht über das Gebiet der Westwindtrift hinaus und verbreiteten sich bis zur Eiskante nach Süden und 
ebenso schien jene Strömung zur Zeit unserer Fahrt auch die Nordgrenze für Phoebetria fuliginosa zu bilden. Ferner ergab sich, dass Pygoscelis antarctica dem Gebiet der Bouvet-Insel angehört, dadieser Pinguin, obwohl ihm Eisberge, die er ja als Transportmittel benutzte, dazu Gelegenheit boten, sich nicht weiter als bis $27^{\circ} \mathrm{O}$. L. nach Osteu von jener Insel entfernte, und dass die DiomedeaArten und auch Procellaria aequinoctialis unter $60^{\circ} \mathrm{O}$. L. weit tiefer nach Süden herabsteigen als unter $10^{\circ} \mathrm{O}$. L.. dass also ihre südliche Verbreitungsgrenze auf einer schrïgen Linie zwischen $46^{\circ} \mathrm{S}$. Br. $10^{\circ}$ O.L. und $59^{\circ} \mathrm{S}$. Br. $65^{\circ} \mathrm{O}$. L. verlief.

Im Norden der Kerguelen wurden bei der stürmischen Fahrt nach St. Paul vom 21. Dezember bis 2. Januar keine Vögel beobachtet. Auf St. Paul und Neu Amsterdam trafen wir dann am 3. und 4. Januar Colonien von Catarractes chrysocome*, die Chun ausführlich schildert, und auf dem Meere fliegend Lestris antarctica, Diomedea exulans und Thalassogeron chlororhynchus an. Am 5. zeigte sich ausser den beiden Albatrossen noch Procellaria aequinoctialis und am 6. fuhren wir an der Grenze der Westwindtrift und der stromlosen Zone des südindischen Stilltengürtels durch eine auf dem ruhigen Wasser sitzende Versammlung von Thalassogeron chlororhyrchus ${ }^{*}$, so dass ich auf beiden Seiten des Schiffes zusammen etwa 300 Vögel zählen konnte. Einige Exemplare des gelbschnäbligen Albatross wurden am 7 . gesehen und ausser ihnen erschien noch eine einsame Procellaria aequinoctialis. Nachdem uns am 8. kein Vogel zu Gesicht gekommen war, zeigte sich am 9. Januar eine Procellaria aequinoctialis als letzter, am weitesten nach Norden vordringender Repräsentant der reichen Vogelwelt der südlichen kalten und gemässigten Zone. Von $29^{\circ}-19^{\circ} \mathrm{S}$. Br. durchfuhren wir vom 10.-14. Januar ein Gebiet, in dem alle Vögel fehlten. In ihm traten die ersten Haifische, Boniten und fliegende Fische auf. Im Süden der Kokos-Inseln wurde dann am 15. der erste indische Tropikvogel Phaeton gesehen; am 16. erschienen zwei und am 17., als wir ganz nahe bei den Kokosinseln fischten, mehrere Tropikvögel beim Schiff, die in der Mittagsstunde zu verschwinden pflegten, während sie am Morgen und Nachmittage eifrig, gelegentlich dicht über dem Wasser hinstreichend, fischten. Hier wurden auch mehrere Exemplare von Sula piscator* und Fregata aquila hoch über dem Schiff ihre Kreise ziehend bemerkt. Fregatte und Sula zeigten sich auch am 18. und 19. Januar, 
während vom 20.-22. Januar bis zu unserer Landung in KöniginEmma-Hafen bei Padang alle Vögel vermisst wurden. An den bewaldeten Höhen, die den vorzüglichen Hafen der Südwestküste Sumatras einschliessen, flog nicht selten ein ziemlich grosser Raubvogel umher, der nicht genau erkannt werden konnte. In Reisfeldern fielen Scharen weisser Reiher auf und an Singkarah-See wurden Eisvögel, Halcyon chloris*, und Irena crinigera* $a^{*}$ erlegt. Ferner sahen wir in geflochtenen Käfigen Tauben und Wachteln und den sprechenden Beo, Eulnbes religiosa; das war Alles, was von Vögeln bei unserem flüchtigen Besuch in Padang zu heobachten war.

Als wir am 30. Januar den Hafen verlassen hatten, um zwischen Sumatra und den Mentawei Inseln zu kreuzen, wurde eine Feenseeschwalbe, Gygis candida, bemerkt. Dann liessen sich keine Vögel blicken bis zum 7. Februar Abends, wo bei der unbewohnten Nicobareninsel Kandul eine grosse Schar sehr kleiner Landvögel ganz niedrig ưber das Schiff hinweg dem Lande zu zog. Sie kamen so plötzlich, dass keiner von ibuen erlegt werden konnte. Auf der Fahrt von den Nicobaren nach Ceylon wurde ein einzelner Tropikvogel gesehen, dann erschien vor Ceylon am 13. ein Schwarm weisser Seeschwalben in der Ferne, und in Colombo selbst zeigte sich ausser einem Raubvogel und Seeschwalben, die über dem Colombosee schwebten, grosse Mengen der schönen schwarzen Glanzkrähe Corvus splendens, die alle Gebusche belebten und sich trotz des lebhaften Treibens auf den Strassen sicher zu fühlen schienen.

Von Colombo steuerten wir dem Suadiva Atoll zu, Unterwegs wurde am 17. Februar ein Tropikwogel beobachtet der ganz ähnlich wie Sula auf seine Beute ins Wasser herabstiess und bei den Suadivainseln selbst flog Gygis candida in grösserer Zahl dicht über dem Wasser. Am folgenden Tage wurde bei der Landung noch ein Tropikvogel gesehen, eine bisher nicht bestimmbare Corvus-Art* und eine Seeschwalbe, Sterna melanauchen*, erlegt. Im Süden dieser Inseln erschienen dann 2 Fregattrögel beim Schiff und am 22. nahe bei den Tschagosin seln trafen wir zum ersten Male Anous stolidus*, die dunklen dummen Seeschwalben an, die in grossen Scharen dicht über dem Wasser hin und her flogen und fischten. Wabrscheinlich verfolgten sie die blauen Copepoden, Pontelliden, die dort in grosser Menge mit dem. Oberflächennetz gefangen werden konnten. 
Die Tölpelseeschwalben, Anous stolidus, nișteten zahlreich auf den Kokospalmen von Diego-Garcia, wo wir am 23. Abends eintrafen. Ausser ihnen wurde noch Anous tenuirostris und Gygis candida* erbeutet, welche letztere sich schön von den dunkelgrünen, die Häuser der Kolonien beschattenden Callophyllumbäumen abhob. Von den bei Ebbe trockenen Corallenriffen erhielten wir Numenius phaeopus", den Brachvogel, und Dromas ardeola", einen kleinen Reiher, die beide ziemlich scheu waren. Endlich gelang es uns dort auch, Foudia madagascariensis", einen kleinen roten Webervogel, und zwei neue Vogelarten zu erlegen, die Reichenow als Homopelia chuni " und Butorides alba. limbatus beschrieb. Die erstere, eine kleine Taube, wurde nur in 2 Exemplaren in dem niedrigen Gebüsch bemerkt, das den Korallensand vom cultivierten, mit Kokospalmen bestandenen Lande trennt. Sie war nicht scheu, suchte sich aber im Laub und dichtem Geäst zu verstecken. Der kleine Reiher, Butorides albolimbatus, schien von einer schrïg uber das Wasser der Lagune heräberragenden Palme Umschau zu halten, als er überrascht wurde. Ein zweites Exemplar wurde später auf dem Strandriff erlegt, wo sich auch noch grössere graue Reiher zeigten, die aber so scheu waren, dass wir nicht auf Schussweite herankommen konnten.

Am 26. Februar nach der Abfahrt von Diego-Garcia kamen 2 Tropikvögel in Sicht. Nachdem dann am 27. alle Vögel ausgeblieben waren, wurde am 28. eine Fregata ariel* erbeutet.

Am 1. und 2. März waren keine Vögel sichtbar, dann aber verrieten am Nachmittag des 3. Sula und Sterna bereits die Annäherung an die Seychellen. Am 4. wurden 2 Tropikvögel geschossen, die nicht aufgefischt werden konnten.

Der Aufenthait auf den Seychellen vom 5.-8. März gab Gelegenheit, etwas von der interessanten Vogelwelt der Inseln kennen zu lernen. Der auffallendste Vogel auf der Hauptinsel $\mathrm{Ma} \mathrm{h}$ é ist Acridotheres tristis, der dort nicht einheimisch ist, sondern eingefübrt sein soll. Gleich beim ersten Spaziergange auf der Insel sahen wir den schwarz und weiss gezeichneten Vogel auf der Weide auf und zwischen Rindern unserem Star ähnlich sich tummeln. Er wird von den Kreolen geschützt, die ihm den Namen „Matin" beigelegt haben, da er am Morgen mit wohlklingendem Ruf die Langschläfer weckt. Als wir am Morgen des 6. März einen Ausflug nach dem Urwalde am Mount Harrison unternahmen, 
hörten wir seinen Ruf von den hohen Bäumen in der Nähe der Häuser. Beim Anstieg in das Gebiet, wo Zimmt, Gewürznelken und Vanille angepflauzt sind, zeigte sich ein Zwergfalk, Hierax(?), auf hohem kahlen Fels am Wege und Nectarinien flogen pfeilschnell vorüber. Zuweilen hörte man auch den Gesang des roten Webers. Im Walde verriet sich die Merle, Ixocincla crassirostris*, durch lautes Geschrei. An bewaldeten Abhängen über tiefen Thälern schwebten graziös in eleganten Wendungen die Pärchen der Feenseeschwalbe, Gygis candida, und hoch oben an den steil aufragenden höchsten Felsspitzen zogen die Tropikvögel schreiend und einander jagend ihre Kreise. Beim Abstieg wurde auf dem Wege am Boden sitzend eine kleine Taube, Geopelia striata", auf Bäumen ein Webervogel, Foudia madagascariensis", und ein Matin, Acridotheres tristis", für unsere Sammlung erlegt.

Am 8. März erhielten wir von der Insel Felicité zwei Tropikvögel, Phaëton lepturus", und eine Warzentaube, Alectroenas pulcherrima*, und von Praslin mehrere Merlen und einen kleinen Reiher, dessen Balg leider nicht präpariert wurde.

Als wir dann nördlich von den Amiranten der afrikanischen Kiuste zusteuerten, wurden am 9. und 10. März Tropikvögel und Fregatten, am 11. Fregatten und Tölpel, Sula cyanops(?), bemerkt. Am 12. fehlten die Vögel; am 13. gelang es einen von mehreren vorüberfliegenden Tölpeln zu schiessen, der als Sula cyanops* bestimmt werden konnte. Am 14. schon in der Nähe von Dar es Salam wurde ein Tropikvogel gesehen und eine Fregatte, Fregata aquila, erlegt.

Am 15. März ankerten wir im Hafen von Dar es Salam, uber dem Raubvögel und eine dunkle Möve schwebten. Dort wurden auf von Mangrove freiem Ebbestrand Tringa suburcuata*, Tringoides hypoleuca* und Charadrius pecuctrius erlegt und Brachvögel gesehen. Im Gebüsch erhielten wir Telephonus senegalus* und Monticola saxatilis*. Sonst zeigten sich noch Geier und Falken, Schildraben, Tauben, Schwalben und Webervögel. Dort wurden die letzten Vögel geschossen.

Als wir dann längs der So maliküste nach Aden fuhren, zeigten sich am 22. März weisse mövenartige Vögel in der Ferne, am 24. wurden schwarze Vögel mit mövenartigem Flug und eine weissköpfige Fregatte gesehen. Dann waren erst wieder am 28. März 4 weisse Vögel (Tropikvögel ?) fern sichtbar und am 29. stiess eine weisse, etwas graue Seeschwalbe (?) tauchend ins 
Wasser. Am 30. Mäz erschien ein Tropikvogel und am 1. April ein kleiner Landvogel in der Nähe des Schiffes.

Am 2. April bei Cap Guardafui und am 4., kurz bevor wir Aden erreichten, wurden noch die letzten Tropikvögel beobachtet. In Aden galt die Tiefseeexpedition für beendet. Dort zeigte sich der erste nordische Vogel, Larus fuscus oder L. marinus. Von der schnellen Heimfahrt, bei der nicht mehr so genaue Beobachtungen äber Vögel gemacht werden konnten, möchte ich noch erwähnen, dass wir etwa in der Mitte des roten Meeres am 9. einen Wiedehopf, Upupa epops, am 12. im Golf von S u ez wieder die dunkle Möve, am 16. angesichts von Kreta unter anderen kleinen Landvögeln eine gelbe Bachstelze, am 17. vor der Strass e von Messin a Möven, einen Falk, Saxicola, zwei Schwalben und eine Lerche antrafen und im Golf von Biscaya am 25. April Larus tridactylus und den ersten Lummen begegneten.

Schon vorher wurde für den atlantischen Ocean a uf den Reichtum der kalten Meere an Arten und Individuen im Gegensatz zu den Meeren der Tropen hingewiesen. Noch auffalliger tritt der Unterschied im indischen Ocean hervor. Dort waren nur 5 Vogelgattungen für das Tropengebiet charakteristisch: der Tropikvogel, die Fregatte, Sula, Anous und Gygis, die, abgesehen von Anous nicht sehr zahlreich, meist in der Nähe des Landes gefunden wurden. Während südlich am $45^{\circ} \mathrm{S}$. Br. kaum ein Tag verging, an dem nicht Vögel beim Schiff erschienen, zeigten sich in den Tropen grosse Lücken, die noch grösser gewesen wären, wenn nicht zahlreiche Inseln auf unserm Wege gelegen hätten. Denn selbst Tropikvögel und Fregatte scheinen sich nur ausnahmsweise weit vom Lande zu entfernen. Den wenigen Vögeln derTropen bietet das ruhige Meer auch in der Nähe der Küsten genügende Nahrung, die Vogelscharen der Antarktis dagegen sind zum Teil darauf angewiesen, weite Strecken zu durchfliegen, und haben oft mit schweren Stürmen zu kämpfen. Daher haben sich dort die ausdauerndsten Flieger herausgebildet. Als solche sind Diomedea exulans und D. melanophrys, Thalassogeron, Phoebetria, Procellaria, Pagodroma, Thalassoeca, Priocella, Priofinus und die Prion-Arten zu nennen. Weniger gut fliegen nach meiner Ansicht Daption capense und Oestrelata mollis, die zwar nur in einem Exemplar beobachtet werden konnte, aber sich in Körperform und Gebaren an die Kaptaube anschliesst. Elegante, aber anscheinend wenig ausdauernde Flieger sind Fregatte, Sula Tropikvögel und die Seeschwalben Sterna, Anous und Gygis, die 
sich in der Regel nicht so weit als jene vom Lande entfernen. Zweimal wurde Sterna völlig erschöpft anf dem Schiff angetroffen und Anous stolidus, die Tölpelseeschwalbe, hat den Namen "Döskopf" von den Seeleuten zum Teil wohl erhalten, weil ihr Mudigkeit und Hunger oft als Dummheit ausgelegt wurde, wenn sie sich auf Schiffen mit der Hand fangen liess. Die langen spitzen Flügel scheinen wohl zu schnellen Wendungen und zum Schweben bei leichter Brise geeignet; aber nicht brauchbar, um bei Sturm die Richtung zu halten. Nicht besonders gewandt erwies sich Larus dominicanus, die sich auch nicht weit von der Küste entfernt, und im Vergleich mit den vorigen müssen Ossifraga gigantea, Phalacrocorax und Lestris als schwerfällig bezeichnet werden. Entsprechend ihrer Beweglichkeit haben sich die Meeresvögel auch ihre Nahrung gewählt. Der Riesensturmvogel und die Raubmöve mästen sich an Cadavern oder leben vom Raube, der Kormoran taucht, um Küstenfische und andere Seetiere zu erbeuten, die Möve nimmt mit den der Oberfläche sich nähernden Fischen, mit Aas, dem Angespül der See und den bei Ebbe am Strande zurückgebliebenen Tieren vorlieb. Die Seeschwalben scheinen über der Oberfläche schwebend Copepoden und die einzigen Insekten des Meeres (Halobates) zu jagen, werden aber auch wohl andere niedere Tiere nicht verschmähen. Bei andauernd stürmischem Wetter auf hoher See müssen sie wahrscheinlich verhungern. Sula und Tropikvogel sind als echte Fischer, die auf erspähte Beute herabstossen, bekannt. DieFregatte zieht raubvogelartig in der Höhe ihre Kreise, streicht aber zuweilen auch niedrig über dem Wasser hin. Wie sie fischt, habe ich nicht beobachten können. Die Sturmvögel und Albatrosse endlich nähren sich von lebenden oder toten an der Oberfläche treibendenTieren. Trotz ihres schnellen Fluges müssen sie gut Umschau halten, da wir im Albatrossmagen selbst von wenig auffallenden Tieren grössere Exemplare fanden, als wir mit unsern Netzen erbeuten konnten.

Diese Beobachtungen über Vorkommen und Lebensweise der Vögel, die ich nach Aufzeichnungen in meinem Tagebuche mitgeteilt habe, werde ich bei der Deutschen Südpolarexpedition vervollständigen und erweitern, da wir auf anderen Wegen als bei der Tiefseeexpedition nach Süden vordringen und von dort zurückkehren werden. Vielleicht giebt mir auch die weitere Beobachtung der Vögel zu andern Jahreszeiten Gelegenheit, meine Anschauungen über das Hinausfliegen der Vögel auf's hohe Meer, 
das in manchen Fällen vom Brutgeschäft beeinflusst sein kann, zu ändern. Jedenfalls hoffe ich nach der Rückkehr von der Südpolarexpedition einen ausführlichen Beitrag zur geographischen Verbreitung der Meeresvögel insbesondere jener der sũdlichen Breiteu liefern zu können.

\section{Zur Ornis des Thales der Drewenz}

(im Weichbilde der Stadt Neumark i. Wpr.).

\section{Von Fritz Braun.}

Mag das Wanderleben eines westpreussischen Schulamtskandidaten auch manche Unannehmlichkeiten mit sich bringen, der Freund der heimischen. Ornis wird diese gern in den Kauf nehmen, bringt ihm doch grade der unstäte Wandel eines modernen Bildungsreisenden grosse, nicht zu unterschätzende Vorteile. Vor anderen lernt er erkennen, dass unser Vaterland auch auf kleinerem Raum durchaus kein einheitliches Gebiet ist, dass dem Forscher in scheinbar recht ähnlichen Gegenden eine gar verschiedene Ornis entgegentritt - eine beherzigenswerte Warnung vor vorschnellem Verallgemeinern. Er begreift, wie wenig damit gesagt ist, eine Vogelart kommt bis hier und dahin vor, er sieht ein, dass die einzelnen Arten inmitten ihres Gebietes hier dichter, dort dünner siedeln und grossen Landstrecken inmitten ihrer Verbreitungszone sogar völlig fehlen, ohne dass man für ihr Verschwinden sogleich einen nahe liegenden Grund anführen könnte. Die Feststellung der verschiedenen Siedelungsdichte einer und derselben Art wird für die genaue Schilderung ihrer Lebensweise, für die Aufklärung der geheimen Zusammenhänge zwischen dem Leben grade dieses Vogels mit der Bodenform, der Vegetation und der Wirtschaftsgeographie oft viel, viel wichtiger sein, als die zufällige Notierung eines vorschnellen Pioniers, der über das Reich seiner Artgenossen hinausstrebte.

Wie angenchm wäre es für uns, wenn wir z. B. für Fringilla serinus eine Karte seiner Siedelungen und seiner Siedelungsdichte besässen. Damit wäre für die kausale Erklärung seiner Nordwanderung wohl die wichtigste Vorarbeit geleistet.

Durch solche Arbeiten kann wegen der Gründlichkeit, die sie verlangen, auch nicht so leicht gesündigt werden, wie bei der isolierten Angabe eines neuen Nistplatzes. In einem der letzten Jahrgänge der "gefiederten Welt" las ich zu meinem Schrecken 